\title{
L'herméneutique biblique de John Lanigan, disciple irlandais de Pietro Tamburini
}

The Biblical Hermeneutics of John Lanigan, An Irish Disciple of Pietro Tamburini

\section{Grazia Grasso}

\section{(2) OpenEdition}

12 Journals

\section{Édition électronique}

URL : https://journals.openedition.org/etudesirlandaises/11602

DOI : 10.4000/etudesirlandaises. 11602

ISSN : 2259-8863

\section{Éditeur}

Presses universitaires de Caen

\section{Édition imprimée}

Date de publication : 30 décembre 2021

Pagination : 7-24

ISBN : 978-2-84133-157-0

ISSN : 0183-973X

\section{Référence électronique}

Grazia Grasso, «L'herméneutique biblique de John Lanigan, disciple irlandais de Pietro Tamburini », Études irlandaises [En ligne], 46-2 | 2021, mis en ligne le 17 décembre 2021, consulté le 03 novembre 2022. URL : http://journals.openedition.org/etudesirlandaises/11602 ; DOI : https://doi.org/10.4000/ etudesirlandaises.11602

\section{(c) $)(1)(2)$}

Creative Commons - Attribution - Pas d'Utilisation Commerciale - Partage dans les Mêmes Conditions 4.0 International - CC BY-NC-SA 4.0

https://creativecommons.org/licenses/by-nc-sa/4.0/ 


\title{
L'herméneutique biblique de John Lanigan, disciple irlandais de Pietro Tamburini
}

\begin{abstract}
Résumé: L'article présente l'œuvre herméneutique de John Lanigan (1758-1825), d'abord disciple du janséniste italien Pietro Tamburini (1737-1827) au Collège irlandais de Rome, puis professeur d'Écritures saintes à Pavie. Chez les Anglo-Saxons, il est considéré plutôt comme un historien. Certains éléments d'originalité de ses travaux d'herméneutique biblique, n'ayant jamais fait l'objet d'études spécifiques, sont ainsi discutés; jugés de grand intérêt, ces sujets seront développés peu après par l'herméneutique philosophique de Schleiermacher, de Dilthey ainsi que de Gadamer.
\end{abstract}

Mots clés: John Lanigan, Bible, jansénisme, Pietro Tamburini, herméneutique biblique.

\begin{abstract}
The article presents the hermeneutical work of John Lanigan (1758-1825), initially a disciple of the Italian Jansenist Pietro Tamburini (1737-1827) at the Irish College in Rome, and subsequently professor of the Holy Scriptures in Pavia. In the English-speaking world, he is considered more of a historian. Certain elements of the originality of his biblical hermeneutic work, never previously the subject of specific studies are discussed. These subjects will then be developed via the philosophical hermeneutics of Schleiermacher, Dilthey and Gadamer.
\end{abstract}

Keywords: John Lanigan, Bible, Jansenism, Pietro Tamburini, biblical hermeneutics.

À la fin du XVIII ${ }^{e}$ siècle, tandis qu'en France les Challe ${ }^{1}$, les Voltaire, les Diderot et les Rousseau se moquaient de la Bible à cause de ses obscurités, contradictions, incohérences et absurdités, du côté des théologiens éclairés, surtout allemands, tels qu'un Eichhorn ${ }^{2}$, la critique scripturaire aboutissait à son accomplissement, éclipsant la lecture allégorique, à la suite de l' «invention critique de la Bible » ${ }^{3}$ par Jean Morin, Louis Meyer ${ }^{4}$, Spinoza, Richard Simon et Jean Astruc.

1. Robert Challe (1659-1721), devenu déiste, est l'auteur des Difficultés sur la religion proposées au père Malebranche, édition critique d'après un manuscrit inédit par Frédéric Deloffre et Melâhat Menemencioglu, Oxford, Voltaire Foundation, 1982.

2. Johann Gottfried Eichhorn (1752-1827), théologien et orientaliste allemand, est un représentant du criticisme biblique. Il écrivit une introduction à l'Ancien Testament et une introduction au Nouveau Testament. Voir Giuseppe D'Alessandro, L'Illuminismo dimenticato: Johann Gottfried Eichhorn (17521827) e il suo tempo, Naples, Liguori, 2000. Voir aussi «Johann Gottfried Eichhorn», Encyclopedia Britannica, en ligne: https://www.britannica.com/biography/Johann-Gottfried-Eichhorn.

3. Voir Pierre Gibert, L'invention critique de la Bible, XV $V^{e}$-XVIII ${ }^{e}$ siècle, Paris, Gallimard (Bibliothèque des histoires), 2010; François Laplanche, Bible, sciences et pouvoirs au XVII siècle, Naples, Bibliopolis, 1997.

4. Jean Morin (1591-1659) est l'auteur des Exercitationes biblicee de 1633. Louis Meyer (1629-1681) a publié la Philosophia sanctae Scripturae Interpres, exercitatio paradoxa en 1666. 
Par ailleurs, en 1758 le pape Benoît XIV, de son côté, publiant la constitution Sollicita ac provida, libéralisait la lecture de la Bible en langue vulgaire; Rome continuait, cependant, à détenir le monopole de ses traductions. En Italie aussi, par conséquent, surtout de 1770 jusqu'à la fin du XVIII ${ }^{\mathrm{e}}$ siècle, l'on assiste à une floraison de nouvelles éditions et de traductions en langue vulgaire, qui montrent un intérêt renouvelé pour les études bibliques ${ }^{5}$.

C'est en effet dans ce contexte européen d'effervescence d'études sur la Bible que le janséniste Pietro Tamburini ${ }^{6}$ appela un jeune prêtre irlandais, John Lanigan (17581825), à enseigner les Saintes Écritures et l'histoire ecclésiastique au séminaire, puis au Collège germano-hongrois, dont il était le directeur à l'Université royale de Pavie ${ }^{7}$.

Peu connu dans le monde francophone, Lanigan est surtout considéré comme un historien dans le monde anglo-saxon car il a rédigé une histoire de l'Église irlandaise, entre 1799 et $1822^{\circ}$. En 1791, Lanigan publia un essai en italien sur

5. Ce qui a donné lieu à une vingtaine d'éditions intégrales de la Bible, tout au long du XVIII siècle. Parmi les plus illustres, la traduction d'Antonio Martini, de 1769 à 1781, ainsi que celles d'IsaacLouis Le Maistre de Sacy, du père Louis de Carrières et de François-Philippe Mésenguy. Isaac-Louis Le Maistre de Sacy publia en 1667 la traduction française du Nouveau Testament à partir du grec, Le Nouveau Testament de Notre Seigneur Jésus Christ, traduit en français selon l'édition Vulgate, avec les différences du Grec, Mons, G. Migeot. Entre 1672 et 1684, il publia une traduction de dix livres supplémentaires de la Bible. Mais la première traduction collaborative de toute l'œuvre en français, depuis la Vulgate, parut en 1696, en trente-deux volumes, intitulée La Sainte Bible contenant l'Ancien et le Nouveau Testament, Paris, G. Desprez; elle est également appelée la Bible de Port-Royal ou de Sacy. Louis de Carrières est célèbre pour ses commentaires littéraux sur tous les livres de la Bible, publiés entre 1701 et 1717, date de son décès. François-Philippe Mésenguy publia l'Abrégé de l'histoire et de la morale de l'Ancien Testament en 1728, Le Nouveau Testament de Notre Seigneur Jésus-Christ en 1729 et l'Abrégé de l'histoire de l'Ancien Testament en dix volumes, entre 1735 et 1753.

6. Pietro Tamburini (Brescia, janvier 1737-Pavie, 1827). Devenu prêtre en 1760, il est appelé par le cardinal Molin, évêque de Brescia, à enseigner au séminaire. Voir la large note biographique en français dans Grazia Grasso, «Pietro Tamburini, chrétien éclairé et lecteur de Bayle», Libertinage et philosophie à l'époque classique (XVI ${ }^{e}$-XVIII ${ }^{e}$ siècle), $\mathrm{n}^{\circ} 15,2018$, Pierre Bayle et les libertins, p. $185-186$, n. 7.

7. Sur l'histoire du Collège germano-hongrois, voir Giulio Guderzo, "Giuseppe II e il Collegio Germanico Ungarico di Pavia ", in Studi in memoria di Mario Abrate, Turin, Università di Torino Istituto di storia economica, 1986, p. 513-533.

8. John Lanigan, An Ecclesiastical History of Ireland from the First Introduction of Christianity among the Irish to the Beginning of the Thirteenth Century, Dublin, D. Graisberry, 1822, 4 vol.; $2^{\mathrm{e}}$ éd. : Dublin - Londres - Édimbourg, J. Cumming - Simpkin and Marshall - R. Cadell, 1829. Donald MacCartney considère l'ouvrage comme « une des meilleures recherches sur l'histoire ecclésiastique durant cette période": "The method that was now being developed by workers like Hardiman, William Monck Mason, Lanigan and the Rev. Charles O'Conor, was a slow but scientific one. John Lanigan, for example, in one of the finest pieces of research on ecclesiastical history during this period, could criticise "natives" like O'Halloran and Keating on the one hand, while on the other, his heavy criticism was aimed at Ledwich. [...] Acton claimed that many of the most conspicuous scientific German historians of the nineteenth century had composed history upon the shoulders of the Italian ecclesiastical historians. Lanigan had been a professor in Pavia, and no doubt had been influenced by these same Italian scholars» (Donald MacCartney, "The Writing of History in Ireland 1800-30", Irish Historical Studies, vol. 10, n 40, septembre 1957, p. 350). Felim Ó Brian avait lui aussi loué le «critical discernement» de Lanigan (Felim Ó Brian, «The Expansion of Irish Christianity to 1200: An Historiographical Survey. Part I», Irish Historical Studies, vol. 3, n 11, 1943, p. 241). Voir l'article de Christopher Korten, «Pietro Tamburini's Jansenist Legacy at the 
l'enseignement de la science biblique, Saggio sulla maniera d'insegnare a' giovani ecclesiastici la scienza de' libri sacri ${ }^{9}$. Il écrivit également un discours en latin ${ }^{10}$, consacré à l'herméneutique biblique, ainsi que les Institutionum Biblicarum. Pars prima qua continetur Historia librorum sacrorum Veteris, et Novi Testamenti ${ }^{11}$.

Les travaux d'herméneutique de Lanigan n'ont jamais fait l'objet d'études spécifiques. Christopher Korten ne les mentionne même pas dans son étude récente sur l'influence de Tamburini sur l'Église d'Irlande ${ }^{12}$.

À la lumière des recherches de Korten, qui contredisent la thèse de William John Fitz-Patrick ${ }^{13}$, son premier biographe, et démontrent l'adhésion consciente et volontaire du jeune Lanigan au jansénisme, par son refus, par exemple, de souscrire aux cinq propositions, on peut s'interroger sur l'influence que le jansénisme a pu avoir dans sa conception de l'interprétation scripturaire et quels sont les éléments d'originalité de son œuvre.

Dans un premier temps, nous nous pencherons sur l'influence que Tamburini a pu exercer sur Lanigan au Collège irlandais d'abord, et à Pavie ensuite, en le motivant à une lecture janséniste de la Bible, à l'appui de sources manuscrites autobiographiques inédites. Dans un second temps, nous analyserons ses œuvres bibliques pour mettre en valeur les éléments d'originalité de son interprétation, qui seront développés plus tard par l'herméneutique philosophique, un nouveau courant, dont le théologien protestant Friedrich Schleiermacher est le père, et Wilhelm Dilthey ainsi que Hans-Georg Gadamer sont les héritiers.

\section{Lanigan, disciple de Tamburini à Rome et à Pavie}

Christopher Korten considère que Lanigan fut véritablement et fortement influencé par les doctrines philo-jansénistes de Tamburini, comme cinq autres élèves du Collège irlandais de Rome. Cette thèse va à l'encontre du portrait de Lanigan

Irish College in Rome and His Influence on the Irish Church», The Catholic Historical Review, vol. 103, n² 2, printemps 2017, p. 293, n. 125. Korten mentionne erronément la même revue de 1943 pour les deux extraits de citation, qui en réalité se trouvent dans deux articles différents. Les traductions en français de l'anglais, de l'italien et du latin ont été réalisées par nos soins.

9. L'ouvrage parut sans indication de date, de lieu d'édition ni de nom d'éditeur. On peut toutefois le dater grâce à sa recension dans le Giornale ecclesiastico di Roma, nº 42, 22 octobre 1791, p. 165-168. Dans sa Biblioteca ecclesiastica e di varia letteratura antica e moderna, Pavie, imprimeur héritier de Galeazzi, 1791, t. II, en particulier p. 2-4, la maison d'édition des ouvrages des jansénistes de Pavie en donne également un excellent résumé, évidemment enthousiaste.

10. John Lanigan, De origine et progressu hermeneuticae sacrae oratio quam in Regio Archigymnasio Ticinensi Joannes Lanigan: Hibernus Cassiliensis, sacrarum litterarum R. Professor, et in Imper. Collegio Germanico-Hungarico earundem et hist. eccl. relector habuit, XI Kal. Iunii an. 1789, Pavie, Galeazzi, 1789. Il s'agit du discours inaugural de son enseignement à Pavie, qu'il prononça en mai 1789.

11. John Lanigan, Institutionum Biblicarum. Pars prima qua continetur Historia librorum sacrorum Veteris, et Novi Testamenti, Pavie, Comini, 1793.

12. Voir Christopher Korten, «Pietro Tamburini's Jansenist Legacy...».

13. William John Fitz-Patrick, Irish Wits and Worthies; Including Dr. Lanigan, His Life and Times, with Glimpses of Stirring Scenes since 1770, Dublin - Londres, J. Duffy, sons, \& co., 1873, p. 55. 
dressé dans sa première biographie ${ }^{14}$ : selon Fitz-Patrick, il n'aurait pas adhéré au mouvement janséniste. Thomas D. F. Barry, qui avait lui aussi étudié à Rome des années plus tard, rendit notamment un jugement en ce sens au sujet du Saggio, que Fitz-Patrick lui avait demandé d'expertiser :

Le sujet est clair, élaboré et assimilé; l'arrangement artistique et efficace; le style me paraît facile, savant et suffisamment éloquent. Mais, mieux encore, il est entièrement exempt de toute trace de jansénisme, que l'on trouvait alors si fréquemment dans les écoles de l'Italie autrichienne ${ }^{15}$.

Pour expliquer le lien de Lanigan avec le jansénisme, il faut revenir un peu en arrière, en 1771, lorsque Tamburini, professeur de théologie au séminaire de Brescia, après avoir publié une longue dissertation janséniste, De Gratia, sur la grâce de Jésus-Christ, est démis de ses fonctions à cause de cette attaque contre les molinistes. Tamburini s'établit alors à Rome ${ }^{16}$. En 1773, alors que la suppression de la Compagnie de Jésus libérait des postes, il fut nommé préfet des études au Collège irlandais où il demeura jusqu'en 1778. Il y avait à Rome à ce moment-là une forte présence du mouvement janséniste; Tamburini connut et fréquenta ses meilleurs représentants.

Il fait également la connaissance de John Lanigan. Né à Cashel en 1758, ce dernier part pour Rome à l'âge de 16 ans avec une lettre de recommandation de l'archevêque de la ville, James Butler, pour poursuivre ses études à l'Irish College, où s'étaient formées plusieurs personnalités irlandaises, notamment des évêques; d'où son surnom de "séminaire des évêques ${ }^{17}$.

Ayant reçu précocement les ordres sacrés, avec une dispense d'âge et un doctorat soutenu conjointement à Rome et à Pavie, Lanigan commença sa carrière de professeur. Conseillé par le cardinal Marefoschi, Lord Protector of Ireland, il

14. William John Fitz-Patrick, Irish Wits and Worthies..., en particulier p. 56, 69, 88, 89, 340, 341.

15. Ibid., p. 56: "The matter is clear, elaborate, and learned; the arrangement artistic and effective; the style seems to me easy, erudite, and sufficiently eloquent. But, better still, it is entirely free from all trace of Jansenism, then so frequently found in the schools of Austrian Italy».

16. Dans son autobiographie inédite, Tamburini décrit sa période romaine, que nous pouvons dater du 6 avril 1773 au 28 novembre 1778, date à laquelle il est nommé professeur de morale à Pavie ([Pietro Tamburini], Autobiografia, manuscrit inédit conservé à la Biblioteca del seminario vescovile de Mantoue, Fondo Labus, X. I. 49). Voir Giovanni Mantese, Pietro Tamburini e il giansenismo bresciano, Milan, Àncora, 1942, p. 72. La date de l'arrivée de Tamburini à Rome de Brescia, 1772, donnée par Christopher Korten dans « Pietro Tamburini’s Jansenist Legacy...» (p. 276), n'est pas correcte. En effet Tamburini lui-même déclare dans une lettre de 1774 au chanoine Bocca être arrivé dans la ville de Rome le Mercredi saint de l'année précédente, c'est-à-dire en 1773, avec son ami Giuseppe Zola. Cette lettre a permis à Giovanni Scarabelli d'établir correctement la date exacte de l'arrivée de Tamburini à Rome, à savoir le 6 avril 1773 (Giovanni Scarabelli, «Le Lettere di Tamburini da Roma al can. Bocca», in Studi in onore di Luigi Fossati, Brescia, Geroldi, 1974, lettre du 26 mars 1774, p. 240 et 244-246).

17. William John Fitz-Patrick, Irish Wits and Worthies..., p. 25: «Seminarium episcoporum». Voir John Meddemmen, «John Lanigan», in Almum studium Papiense. Storia dell'Università di Pavia, vol. II, Dall'età austriaca alla nuova Italia, t. I, L'età austriaca e napoleonica, Dario Mantovani (dir.), Milan, Cisalpino, Istituto Editoriale Universitario, 2015, p. 219-222. 
accepte de fait de suivre Tamburini à Pavie ${ }^{18}$ où, de 1778 à $1795^{19}$, il enseigna alors l'histoire sacrée et l'herméneutique biblique à la Faculté de théologie de l'universitée ${ }^{20}$.

Lorsque Lanigan arriva à Rome, le Collège irlandais, après une période brillante, traversait un moment difficile en raison de la suppression de l'ordre des Jésuites qui, jusque-là, en assuraient la direction. Le biographe de Lanigan juge que cette suppression fut l'erreur la plus grave du pape Ganganelli ${ }^{21}$, ce qui révèle sa sympathie pour l'ordre supprimé. La formation de Lanigan fut donc marquée par les années passées au Collège, sous la direction de prêtres séculiers, parmi lesquels Tamburini.

En général, les historiens irlandais semblent avoir volontairement nié la portée de l'influence des doctrines jansénistes sur les élèves du Collège irlandais de Rome. Michael O'Riordan, ancien recteur et historien de ce Collège ${ }^{22}$, écrivait que Tamburini n'avait certainement pas influencé ses étudiants car, à Rome, il ne faisait pas état de ses convictions personnelles devant ses élèves, mais se limitait à enseigner les doctrines officielles. À l'occasion de la célébration du onzième centenaire de la fondation de l'université de Pavie, à l'été 1925, le sénat académique décida de poser une plaque commémorative en l'honneur de Lanigan. La plaque fut enfin placée le 5 novembre de la même année, pendant l'inauguration solennelle de l'année académique; Timothy Corcoran, professeur à l'université de Dublin et invité à la cérémonie, rédigea ensuite un article relatant l'événement, très élogieux à l'égard de Lanigan, et qui l'exemptait aussi de toute contamination janséniste ${ }^{23}$. Il en vint même à insinuer que, lors du Synode de Pistoia ${ }^{24}$, les rapports entre Tamburini

18. Tamburini quitta Rome en 1778 , pour enseigner à Pavie, où l'impératrice Marie-Thérèse avait fait refondre les études universitaires dans le cadre d'un vaste projet de réformes ecclésiastiques. En 1782, Joseph II déplaça de Rome à Pavie le Collège germano-hongrois, qui fut confié au professeur brescian. Lanigan regretta le départ de Tamburini de Rome pour Pavie et supplia son maître de continuer à le diriger, à distance, de manière épistolaire. Mais les lettres échangées entre Lanigan et Tamburini, contenant un jugement négatif sur les capacités du recteur Luigi Cuccagni à diriger les étudiants et sur sa pédanterie, furent interceptées, et, dès lors, les rapports entre Tamburini et Cuccagni se dégradèrent. Voir [Pietro Tamburini], Autobiografia, folio 41 verso, folio 42 recto. Sur l'histoire du Collège irlandais de Rome, voir The Irish College, Rome and Its World, Dáire Keogh, Albert McDonnell (dir.), Dublin, Four Courts Press, 2008.

19. Voir la fiche bio-bibliographique dans Memorie e documenti per la storia dell'Università di Pavia e degli uomini più illustri che v'insegnarono, Pavie, Bizzoni, 1878, vol. I, Serie di rettori e professori con annotazioni, p. 580. Sur la Faculté de théologie, voir Marco Bernuzzi, La Facoltà teologica dell'Università di Pavia nel periodo delle riforme (1767-1797), Milan, Cisalpino, Istituto Editoriale Universitario (Fonti e studi per la storia dell'Università di Pavia), 1982, p. 5.

20. On l'appelait le "professeur garçon" à cause de son jeune âge (William John Fitz-Patrick, Irish Wits and Worthies..., p. 40 : «the boy professor»).

21. Ibid., p. 28.

22. Voir Christopher Korten, «Pietro Tamburini’s Jansenist's Legacy...», p. 272, n. 3 et 4.

23. Timothy Corcoran, «Ireland and Pavia: 825-1925», Studies: An Irish Quarterly Review, vol. 14, $\mathrm{n}^{\circ} 56$, décembre 1925 , p. 595-610, en particulier p. 597-598.

24. Pietro Stella, La bolla Auctorem fidei (1794) nella storia dell'ultramontanismo: saggio introduttivo e documenti, Rome, LAS, 1995. Voir Il Sinodo di Pistoia del 1786: Atti del Convegno internazionale per il secondo centenario: Pistoia-Prato, 25-27 settembre 1986, Claudio Lamioni (dir.), Rome, Herder, 1991. Voir aussi Grazia Grasso, «La plus forte réaction italienne à la bulle Auctorem Fidei: les Riflessioni in difesa di M.r Scipione de Ricci e del suo Sinodo di Pistoja sopra la costituzione Auctorem Fidei», 
et Lanigan étaient devenus conflictuels, et que c'est pour cette raison que Lanigan aurait décliné l'invitation à ce synode. Il est évident qu'on tentait de faire disparaître toute trace de prétendue hétérodoxie du curriculum d'un illustre confrère ${ }^{25}$.

Tamburini appréciait le rôle de la raison dans la recherche de la vérité et dans l'enseignement. Il ne demandait pas à ses disciples de se fier aveuglément et passivement à ses leçons. Les cours dispensés étaient certainement caractérisés par une méthode interactive, qui favorisait la discussion, l'échange des idées et valorisait l'esprit critique. Nous apprenons de Tamburini lui-même qu'il sollicitait des élèves du Collège irlandais l'exercice de la liberté de pensée par des débats et par la lecture de livres, peut-être même ceux qui étaient censurés ou prohibés. Cette méthode était contraire, d'après Tamburini, aux procédures précédentes, mises en place par les jésuites, qui ne demandaient aux élèves qu'une obéissance aveugle et instillaient dans les consciences des scrupules infinis:

Ces jeunes de bien, pleins de talents et d'envie d'étudier, avaient été dressés par les jésuites de manière mesquine. Toute leur occupation consistait à lire les textes, qui étaient dictés à l'école, et à les apprendre par cœur. Tout leur art s'exprimait en syllogismes et en disputes continuelles. Aucun intérêt pour les bons livres, au contraire, il était défendu de les lire $[\ldots]^{26}$.

in Du jansénisme au modernisme: la bulle "Auctorem fidei", 1794, pivot du magistère romain, JeanBaptiste Amadieu, Simon Icard (dir.), Paris, Beauchesne (Théologie historique), 2020, p. 135-152.

25. Un autre élève de Tamburini, plus célèbre et plus noble, avait lui aussi fait l'objet d'un travail de mémoire visant à minimiser son adhésion au jansénisme: le comte autrichien Thaddeus Trautmansdorf, devenu plus tard cardinal en Autriche, coauteur du De tolerantia ecclesiastica et civili de Tamburini, et qui venait, quant à lui, du Collège germano-hongrois de Rome, transféré par Joseph II à Pavie. Voir Grazia Grasso, «I veri autori del De tolerantia ecclesiastica et civili (Pavia, 1783) ", Rivista di storia del cristianesimo, vol. 15, n 1, 2018, p. 129-146; Grazia Grasso, «La liberté religieuse dans le traité "De la tolérance ecclésiastique et civile" de Pietro Tamburini et de Thaddeus Trautmansdorf (Paris, 1796): de la théologie janséniste de la grâce aux droits civils ", in Port-Royal au XIX ${ }^{e}$ siècle, Paris, Société des Amis de Port-Royal (Chroniques de Port-Royal; 65), 2015, p. 77-94; Grazia Grasso, «Pietro Tamburini difensore della Chiesa di Utrecht. Ruolo dell'edizione belga del De tolerantia, Gand, 1784 ", Revue d'histoire ecclésiastique, vol. 114, n 1-2, 2019, p. 265-288.

26. [Pietro Tamburini], Autobiografia, folio 17 verso: «Que’ bravi giovani pieni di talento, e di voglia di studiare erano stati allevati da gesuiti meschinamente. Tutta la loro occupazione consisteva nel leggere gli scritti, che li si dettavano in scuola, ed impararli a memoria. Tutta l'arte stava nel sillogizzare, e disputare eternamente. Niun gusto de' buoni libri, anzi era vietato il leggerli [...]». Le passage continue, affirmant, qu'au contraire, Tamburini «a poco a poco instillo ad essi il buon gusto. Gli diede i migliori libri da leggere e temperò l'ardente voglia della disputa scolastica. Gli avvezzò ad un esame analitico per calcolare la forza delle prove, e delle obiezioni. [...] Si senti tutto commosso al vedere i più bravi giovani quasi istupiditi, e divenuti quali meri automi per parte della coscienza, che $i$ Gesuiti giuocavano a loro piacere tenendoli schiavi con una cieca ubbidienza, che loro si rendea abituale col farli impazzir per gli scrupoli» (ibid., folio 18 recto); "Cosi facilmente riuscivano a dominare la gioventù con assoluto dispotismo» (ibid., folio 18 verso) [ [ [...] [Tamburini] instilla peu à peu en eux le bon goût. Il leur donna les meilleurs livres à lire et tempéra l'ardente envie de la dispute scolastique. Il les habitua à un examen analytique pour calculer la force des épreuves et des objections. Ainsi, il introduisit auprès de ces jeunes de bien la bonne méthode, de laquelle ils tirèrent en peu de temps un très grand profit, et regrettèrent le temps perdu dans ces disputes scolastiques où ils étaient contraints dans les écoles des jésuites. [...] Il fut tout ému à la vue des meilleurs jeunes rendus presque stupides et tels de pures automates à cause de leur conscience, et 
Mais surtout, selon Tamburini, les jésuites interdisaient aux élèves la lecture directe de la Bible ${ }^{27}$. Cela témoigne qu'une forme de lecture clandestine de la Bible en langue vulgaire était pratiquée à Rome, au moins par des jeunes clercs des séminaires romains. Cela pourrait atténuer, peut-être, la thèse d'origine luthérienne ${ }^{28}$, devenue presque une vulgate, soutenant que l'Italie se caractérisait par une très modeste diffusion de la Bible, et qui ne semble plus correspondre aux recherches archivistiques les plus récentes, surtout après la publication du Panorama delle traduzioni bibliche in volgare prima del Concilio di Trento d'Edoardo Barbieri ${ }^{29}$. Comme certaines études menées dans les dernières décennies l'ont montré, la diffusion de la Bible, dès la première modernité, aurait même été en Italie plus importante qu'ailleurs ${ }^{30}$.

$\mathrm{Au}$ XVIII ${ }^{\mathrm{e}}$ siècle, alors que la production de livres religieux diminuait partout en Europe, on imprimait cependant une grande quantité de bibles: «Jamais depuis Luther autant de traductions, de commentaires ${ }^{31}$. La lecture directe et intégrale de la Bible était toutefois réservée à une élite, surtout constituée d'ecclésiastiques et de moines, dans la mesure où une grande partie de la population ne savait pas lire à la fin du XVIII siècle.

Concernant l'intérêt pour l'étude de la Bible dans les langues originales et ses traductions dans les langues nationales, au XVIII ${ }^{\mathrm{e}}$ siècle, chez les catholiques, Yves-Claude Gélébart, dans le livre dirigé par Yvon Belaval et Dominique Bourel, Le siècle des Lumières et la Bible, observe qu'une

[...] révolution des esprits a été suscitée en partie par les Lumières venues de l'Allemagne du Nord, en majorité protestante, mais aussi par divers mouvements de réforme venus d'Autriche et d'Italie. Relevant le défi des Lumières, la pensée catholique s'impose un délestage du lourd héritage baroque et retrouve ses sources les plus authentiques: la Bible et les Pères de l'Église: il en découle une profonde réforme des études, ainsi qu'un renouveau de la piété, où la Bible occupe la place centrale ${ }^{32}$.

que les jésuites manipulaient à leur guise en les maintenant esclaves par une obéissance aveugle, qui leur était devenue habituelle, et en les faisant devenir fous à cause des scrupules »; "Ainsi ils réussissaient à dominer facilement la jeunesse par un despotisme absolu»].

27. Ibid., folio 17 verso: «[aux étudiants:] gli si facea persino scrupolo anzi la proibizione di leggere la Sacra Scrittura» [ "on les faisait se sentir coupables; de plus il leur était interdit de lire les Saintes Écritures"].

28. Voir Emidio Campi, Mariano Delgado, «Bibles in Italian and Spanish», in The New Cambridge History of the Bible, vol. III, From 1450 to 1750, Euan Cameron (dir.), Cambridge, Cambridge University Press, 2016, p. 358-381; cité par Erminia Ardissino dans Gli Italiani e la Bibbia nella prima età moderna, Erminia Ardissino, Élise Boillet (dir.), Turnhout, Brepols (Études renaissantes; 28), 2018, p. 9, n. 6.

29. Partie I publiée dans Folia theologica et canonica, vol. 8, 1997, p. 169-197; partie II dans Folia theologica et canonica, vol. 9, 1998, p. 89-110; réimpression: Milan, CRELEB-Università Cattolica - CUSL, 2011, p. 1-60.

30. Voir Gli Italiani e la Bibbia nella prima età moderna.

31. Yvon Belaval, Dominique Bourel, «Introduction: la Bible en pleine lumière», in Le siècle des Lumières et la Bible, Yvon Belaval, Dominique Bourel (dir.), Paris, Beauchesne (Bible de tous les temps; 7), 1986, p. 13.

32. Yves-Claude Gélébart, «La Bible dans l'Aufklärung catholique», in Le siècle des Lumières et la Bible, p. 563. 
Il est d'ailleurs également vrai qu'avant ce siècle «[...] les autorités religieuses n'étaient guère favorables à une généralisation de la lecture de la Bible en langue populaire. On traduisait donc d'après la Vulgate, on en faisait des paraphrases, ou bien l'on recueillait des Histoires saintes et exemplaires, mais on n'osait pas traduire la Bible en bloc, hébraïque ou grecque ${ }^{33}$. «C'est au siècle des Lumières que les catholiques allemands - et autrichiens - ont appris à connaître et à aimer la Bible, à en faire leur livre de chevet, comme jadis les légendes des saints $»^{34}$.

Tamburini, qui réfléchissait à cette époque à la place que la Bible et les Pères de l'Église devaient avoir en théologie, a pu influencer Lanigan en attisant son intérêt pour l'étude des Écritures ${ }^{35}$, ce qui pouvait correspondre à son rôle de conseiller aux études.

À Pavie, sous l'œil attentif de son maître, Lanigan, refusant que les cours d'Écriture sainte se réduisent à de simples commentaires et insistant sur la nécessité de connaître les disciplines profanes avant d'aborder les études bibliques, exposait dans le Saggio sulla maniera d'insegnare a' giovani ecclesiastici la scienza de' libri sacri sa méthode pour expliquer la Bible aux étudiants qui se destinaient à la carrière ecclésiastique et, plus généralement, sa conception de l'interprétation scripturaire. Il abordait là un sujet controversé à son époque, en raison du développement de la critique biblique ${ }^{36}$.

33. Yves-Claude Gélébart, «La Bible dans l'Aufklärung catholique», p. 569-570.

34. Ibid., p. 574. Cependant, il ne faut pas oublier que les jansénistes du XVII ${ }^{\mathrm{e}}$ siècle avaient déjà également insisté sur la lecture de la Bible et contribué à sa divulgation grâce aux traductions; pour la Bible de Port-Royal, voir le compte rendu de Philippe Sellier, «Bernard Chédozeau, PortRoyal et la Bible. I. Un siècle d'or de la Bible en France (1650-1708) ", Publications électroniques de Port-Royal, série 2010, en ligne: http://www.amisdeportroyal.org/societe/index.php/2011/01/06/ bernard-chedozeau-port-royal-et-la.

35. Antonio Quacquarelli exprime une observation intéressante sur le rapport entre Tamburini et la Bible dans Il "De praescriptione haereticorum" di Tertulliano nella polemica giansenista di Pietro Tamburini, Bari, Adriatica, 1953, p. 115, 116 et 118: «É dunque [quella di Tamburini] la crisi di un'anima posta ai margini tra il cattolicesimo e il protestantesimo [...]. Egli è della tesi della Bibbia tesaurizzata al massimo come testo di morale e di illuminazione religiosa. Come $i$ protestanti la vuole divulgata e resa accessibile mediante traduzioni. Viene cosi a interferire con l'orientamento opposto dei gesuiti, che non solo non ne vogliono la traduzione, ma l'additano come fonte da attingersi con grande precauzione dai profani, in quanto l'interpretazione senza il magistero è sempre potenzialmente causa di equivoci e di fraintendimenti. Egli invece crede che letta più direttamente possibile la Bibbia sia sempre più di significato facile ed educativo. [...] $\dot{E}$ cosi portato lui cattolico, cattolicissimo a battere le orme dei protestanti» [ "Il s'agit donc de la crise d'une âme [celle de Tamburini] qui est à la frontière entre catholicisme et protestantisme [...]. Il fait partie de ceux qui pensent que la Bible doit être valorisée au maximum comme texte de morale et d'illumination religieuse. Comme les protestants, il souhaite qu'elle soit divulguée et rendue accessible par des traductions. Il en vient ainsi à interférer avec les jésuites, qui eux, non seulement ne veulent pas de traduction, mais la pointent du doigt comme source à laquelle les profanes doivent puiser avec la plus grande précaution; car l'interprétation sans le magistère est toujours potentiellement cause d'équivoques et de malentendus. Il croit en revanche que, lue le plus directement possible, elle est de plus en plus facile à comprendre et éducative. [...] Il est ainsi conduit, lui catholique, même très catholique, à marcher sur les traces des protestants»].

36. Pierre Gibert, L'invention critique de la Bible, $X V^{e}-X V I I I^{e}$ siècle. 
En 1793, Lanigan publia son magnum opus, Institutionum Biblicarum. Pars prima qua continetur Historia librorum sacrorum Veteris, et Novi Testamenti, « une œuvre pleine d'érudition et adressée aux érudits ${ }^{37}$, selon Giuseppe Maria Pujati ${ }^{38}$, ancien professeur de Saintes Écritures à l'université de Padoue. Mais la seconde partie de l'ouvrage ne parut jamais. Pour Fitz-Patrick, le titre de l'ouvrage montre que Lanigan avait l'intention de préparer un second volume, et peut-être même d'autres, afin de mener à bien son projet ${ }^{39}$.

Lors de la rédaction de An Introduction to the Critical Study and Knowledge of the Holy Scriptures ${ }^{40}$, le bibliste Thomas Hartwell Horne chercha pendant six ans l'ouvrage de Lanigan, qui avait disparu. L'œuvre était introuvable en Angleterre, tandis que des rumeurs se répandaient sur sa possible destruction, suite à l'échec de son auteur à obtenir l'imprimatur. Fitz-Patrick attribue la disparition du livre de Lanigan aux jalousies malveillantes de ses collègues ${ }^{41}$.

Cependant, Tamburini apporte une explication plus simple dans son autobiographie, lorsqu'il fait mention d'un accident arrivé à la seconde partie du manuscrit de Lanigan: il rapporte que le jeune homme, en quittant Pavie pour

37. Giuseppe Maria Pujati, Saggio di ermeneutica sacra, Venise, Giuseppe Piccotti, 1819, p. V: «Questa ché dotta di molto e fatta pei dotti anzi che no [...]".

38. Giuseppe Maria Pujati (1723-1824), d'origine frioulane, fut d'abord membre de l'ordre des Pères Somascans lorsqu'il enseigna à Brescia et, pendant sept ans, au Collège clémentin de Rome. Il passa ensuite à l'ordre bénédictin dans le monastère de Subiaco. À Rome, il fréquenta le cercle janséniste et resta en correspondance avec Tamburini, avec qui il fut très ami. Les capacités exceptionnelles de Lanigan ont été remarquées par William John Fitz-Patrick, son premier biographe qui, dans Irish Wits and Worthies..., p. 1, le présentait comme "the eminent ecclesiastical historian of Ireland» [«l'éminent historien ecclésiastique de l'Irlande»]. Tamburini, quant à lui, appréciait plutôt l'intelligence et la connaissance des langues anciennes et modernes dont faisait preuve celui qui avait été son plus brillant disciple au Collège irlandais de Rome, et qu'il présentera plus tard, dans son autobiographie inédite, comme doué «d'un talent extraordinaire, et d'une admirable habileté dans toutes les sciences» ([Pietro Tamburini], Autobiografia, folio 41 verso). Anthony Blake (1758-1787), un élève du même Collège, devenu évêque par la suite, écrivit également au sujet de Lanigan: "I can say with certainty that his talents and extraordinary acquirements, as well as his amiable natural disposition, gained for him the love and admiration of all who knew him" [ "Je puis dire avec certitude que ses talents et sa culture extraordinaire, ainsi que son amabilité naturelle, lui procurèrent l'amour et l'admiration de tous ceux qui le connurent »] (William John Fitz-Patrick, Irish Wits and Worthies..., p. 37).

39. William John Fitz-Patrick, Irish Wits and Worthies..., p. 44: «This learned work of Dr. Lanigan, comprised in one volume, seems to have formed only a portion of his plan; for it is evident he desired to prepare another volume, if not more, to complete his design» [ "Ce travail érudit du $\mathrm{D}^{\mathrm{r}}$ Lanigan, composé d'un seul volume, semble avoir formé seulement une partie de son plan; pour cela il est évident qu'il désirait préparer un autre volume, si non plus, pour accomplir son dessein»].

40. La $7^{\mathrm{e}}$ édition contient une mention du livre de Lanigan: "The second part of this work has never appeared; nor has the writer of these pages been able to obtain the sight even of a copy of the first portion. He has been informed, that it was suppressed in Italy [...]» [ "La seconde partie de cette œuvre n'est jamais apparue. L'auteur de ces pages n'a pas eu non plus la possibilité de voir un exemplaire de la première partie. Il a été informé de sa suppression en Italie [...]»] (Thomas Hartwell Horne, An Introduction to the Critical Study and Knowledge of the Holy Scriptures, $7^{\mathrm{e}}$ éd., Londres - Édimbourg - Dublin, Cadell - Blackwood - Milliken, 1834, t. II, partie II, p. 154). La réédition à Philadelphie, Desilver, Thomas \& Co. en 1836 contient la même mention à la page 73 .

41. Voir William John Fitz-Patrick, Irish Wits and Worthies..., p. 42 sq. 
retourner en Irlande, remit le manuscrit de la seconde partie, contenant ses cours d'herméneutique biblique, à l'imprimeur Agnelli de Lugano, mais qu'un "infortunio», un "accident», serait arrivé à ces copies:

Rien ne manquait donc pour parfaire l'instruction des élèves germaniques, surtout parce que, peu après, il [Tamburini] réussit à faire venir au Collège un talent extraordinaire, M. Giovanni Lanigan, docteur irlandais, qui venait de Rome pour retourner dans sa patrie; il fut arrêté à Pavie, accueilli au Collège, et après avoir été relevé, par Rome et grâce à l'intervention de la Cour de Vienne, de son serment de retourner aux Missions d'Irlande, il fut nommé répétiteur au Collège germanique, puis professeur d'herméneutique à l'Université royale où il déploya ses divers talents et laissa espérer de plus grands progrès, qui furent ensuite abrégés par son retour en sa patrie, à cause des circonstances du temps. Son premier volume préliminaire à l'herméneutique imprimé à Pavie peut être vu comme une preuve de la profondeur de son érudition, et de son savoir singulier en un âge aussi juvénile. On peut regretter qu'à cause d'un brusque accident ses belles leçons d'herméneutiques se soient perdues, alors que, en quittant l'Italie, il les avait déposées entre les mains de l'éditeur Agnelli, à Lugano ${ }^{42}$.

Le récit de cet événement a probablement généré une confusion ou alimenté des rumeurs sur la mise à l'index de l'œuvre entière de Lanigan, qui ne pouvait pas la défendre car déjà décédé.

Un résumé de la première partie, consacrée à l'Ancien Testament, fut également publié dans la Monthly Review ${ }^{43}$.

En conclusion, Tamburini a certainement influencé la formation de Lanigan; celui-ci était son élève préféré, et très apprécié par Tamburini pour ses qualités intellectuelles et humaines, au point de tout mettre en œuvre pour l'appeler à Pavie, à ses côtés, afin de dispenser des cours bibliques.

\section{Une lecture janséniste de la Bible?}

Dans les milieux philo-jansénistes lombards, vénitiens et romains des dernières décennies du XVIII ${ }^{\mathrm{e}}$ siècle et des premières du XIX' ${ }^{\mathrm{e}}$, caractérisées par des bouleversements

42. [Pietro Tamburini], Autobiografia, folio 57 verso, folio 58 recto: «Onde nulla mancava a rendere perfetta la istruzione degli alunni germanici, massimamente, che poco dopo riusci al Tamburini di acquistare al Collegio un ingegno straordinario il Sig.r Don Giovanni Lanigan Irlandese, che venendo da Roma, per andarsene alla Patria fu dal Tamburini, ch'era stato di lui Maestro in Roma, fermato in Pavia, accolto in Collegio, e coll'intervento della Corte di Vienna ottenuta da Roma l'assoluzione dal giuramento prestato di ritornarsene alle Missioni d'Irlanda, fu eletto ripetitore del Collegio Germanico, e poi Professore d'ermeneutica sulla Regia Università dove spiegô i suoi vari talenti, e diede speranze dei più fausti progressi, che furono poi truncate col suo ritorno alla patria per le circostanze de' tempi. Il suo primo tomo preliminare all'ermeneutica stampato in Pavia puô essere un saggio della sua profonda erudizione, e sapere singolare nell'età sua giovanile. Peccato, che per repentino infortunio si sieno perdute le sue belle lezioni d'Ermeneutica, ch'egli nel partir dall'Italia depose in Lugano in mano dell'Agnelli stampatore".

43. Monthly Review, vol. XXII, 1796, p. 552-554. 
révolutionnaires et par des guerres, plusieurs prêtres se distinguaient par leur rigueur morale, leur désir de changements radicaux dans l'Église et leur millénarisme ${ }^{44}$. Ils souhaitaient rendre à l'Église sa pureté originelle et son organisation primitive, à la lumière d'une relecture millénariste de la Bible qu'ils trouvaient dans les œuvres des jansénistes français : la conversion des Juifs, remplaçant les chrétiens apostats issus de la gentilité, devait offrir le temps béni d'une nouvelle jeunesse à l'Église avant le retour du Christ à la fin des temps.

Parmi ces prêtres philo-jansénistes, qui font partie du même réseau culturel et amical, Giuseppe Maria Pujati mentionne l'œuvre de Lanigan. Moine bénédictin, ami et collègue de Tamburini à Rome, Pujati publie en 1819 un Saggio di ermeneutica sacra pour donner aux étudiants des séminaires des outils interprétatifs modernes. Comme Tamburini, il fréquentait le cercle romain dit l' «Archetto ${ }^{45}$ et avait, peut-être, eu l'occasion de connaître Lanigan à Rome. Pujati écrivit notamment un essai sur le millénarisme, intitulé Esame della opinione da' moderni millenari cattolici del regno visibile in terra di Gesù Cristo ${ }^{46}$. Ce sujet n'était pas étranger à Lanigan qui y fait référence dans son Saggio. Il y est convaincu que la nation juive sera convertie un jour et reconnaîtra son Sauveur:

Ces recherches sont devenues de nos jours [1791] plus intéressantes que d'habitude en raison de la controverse entre biblistes et théologiens concernant le temps de la conversion du peuple juif: si celle-ci arrivera seulement à la fin des siècles, ou si elle précédera cette grande catastrophe ${ }^{47}$.

44. Voir [Giovanni Girolamo Calepio], Del ritorno degli ebrei e di ciò che vi ha da porgere occasione, Brescia, Rizzardi, 1772; Pietro Stella, Il giansenismo in Italia, Rome, Edizioni di Storia e Letteratura, 2006, 3 vol.; Marina Caffiero, «Roma nel Settecento tra politica e religione. Dibattito storiografico e nuovi approcci », Dimensioni e problemi della ricerca storica, $\mathrm{n}^{\circ} 2$ 2, 2000, p. 81-100; Marina Caffiero, «Rivoluzione e millennio. Tematiche millenaristiche in Italia nel periodo rivoluzionario», Critica storica, vol. 24, n 4,1987 , p. 584-602.

45. Implanté à Rome suite à l'installation d'un groupe d'ecclésiastiques dans les vingt dernières années du XVII ${ }^{\mathrm{e}}$ siècle, le jansénisme se développa dans la capitale du catholicisme à partir de la moitié du XVIII ${ }^{e}$ siècle, surtout grâce à monseigneur Giovanni Bottari. Le «Circolo » janséniste romain, dit «dell'Archetto», peut être considéré comme le lieu de naissance aussi du jansénisme toscan, car de nombreux intellectuels toscans s'y réunissaient régulièrement chez le comte Fabio de' Vecchi. Faisaient notamment partie de cette association: Giovanni Gaetano Bottari, Pier Francesco Foggini, Scipione de' Ricci, Cristoforo Amaduzzi, Paolo del Mare et Francesco Alpruni (voir Romana Palozzi, "Mons. Giovanni Bottari e il circolo dei giansenisti romani", Annali della R. Scuola Normale Superiore di Pisa. Lettere, Storia e Filosofia, série II, vol. 10, n 1/2, 1941, p. 70-90 et n 3, 1941, p. 199-220). Ils avaient l'habitude de lire et commenter les Pères de l'Église. Tamburini fréquenta aussi l' "Archetto " et se chargea de présenter à cet auditoire cultivé et sensible aux thèmes de la réforme de l'Église le livre des Prescriptions de Tertullien, ainsi que d'autres œuvres de saint Justin et d'Origène.

46. Giuseppe Maria Pujati, Esame della opinione da' moderni millenari cattolici del regno visibile in terra di Gesù Cristo, Venise, Francesco Andreola, 1814.

47. John Lanigan, Saggio sulla maniera d'insegnare a' giovani ecclesiastici la scienza de' libri sacri, p. 106: "Queste ricerche sono diventate ai nostri giorni più interessanti del solito per ragione della controversia, che vi è tra gl'Interpreti, e $i$ Teologi, se la conversione del popolo Ebreo avrà da succedere solamente nel finire dei secoli, o se precederà di lungo tempo cotesta grande catastrofe». 
Lanigan reste prudent, aussi bien sur l'imminence de cette conversion que sur l'interprétation de certaines prophéties bibliques, ne décidant pas clairement si elles concernent l'Israël spirituel, c'est-à-dire l'Église, ou l'Israël charnel. Lanigan est persuadé que certaines prophéties bibliques ne sont pas encore accomplies, mais il conseille d' "adopter une attitude de grande prudence lors de leur explication» :

Il ne faut pas prétendre qu'un professeur puisse dicter des règles pour résoudre toutes les difficultés, puisque pas même les commentateurs ne peuvent, malgré leurs efforts, pénétrer le sens de toutes ces prédictions, qui doivent encore s'accomplir. Le Code des divines révélations comprend encore beaucoup d'autres temps; c'est pourquoi plus la fin des temps approche, plus l'objet et le levé des prophéties se développent. [...] Il y en a encore d'autres, cependant, qui restent couvertes par un voile sacré, qui ne pourra pas être entièrement soulevé, jusqu'au temps où les évènements annoncés arriveront ${ }^{48}$.

Sur la question de la conversion des Juifs et des prophéties bibliques dont il faut encore attendre l'accomplissement, Lanigan semble se situer dans la lignée du jansénisme millénariste et figuriste de Duguet et d'Étemare, quoiqu'avec plus de prudence que son maître Tamburini ${ }^{49}$.

Pour résister à la marée de livres venus d'au-delà des Alpes, qui remettaient en question l'inspiration divine des Écritures et les combattaient ouvertement, en raison de leurs nombreuses contradictions et obscurités, Giuseppe Maria Pujati met en avant trois œuvres italiennes traitant d'herméneutique biblique: Elementa sacrae hermeneuticae, seu Institutiones ad intelligentiam, sacrarum scripturarum de Giacinto Arizzarra ${ }^{50}$, les ouvrages de Lanigan et la traduction italienne de l'ouvrage de Duguet, Regole per intendere le Sante Scritture ${ }^{51}$. Lorsque Pujati écrit, l'Église vient d'autoriser les traductions de la Bible en langue italienne et la publication de nouvelles éditions. L'œuvre d'herméneutique biblique de Lanigan apparaît comme une autorité dans le contexte italien de l'époque. Comme Arizzarra et Pujati lui-même, il cherche à établir les règles et les principes nécessaires pour l'intelligence du texte biblique. Ces augustiniens convaincus, qui entendaient fonder leur herméneutique biblique sur

48. John Lanigan, Saggio..., p. 124-125: «[...] non si dee pretendere che un Professore possa dar regole, coll'ajuto delle quali esse tutte si sciolgano, giacché nemmeno i Commentatori, per quanti sforzi facciano, possono arrivare a penetrare il senso di tante predizioni, che rimangono ancora da compiersi. Il Codice delle divine rivelazioni comprende tutti i tempi; ond'è che quanto più si avvicina il finire dei secoli, tanto più si sviluppano lo scopo e l'oggetto delle Profezie. [...] Ve ne sono però molte ancora, le quali restano coperte da un sacro velo, il quale non si potrà levare interamente, finché verrà il tempo, in cui succederanno gli avvenimenti predetti».

49. Voir Grazia Grasso, «La perfection de la vie chrétienne chez Pietro Tamburini», in Port-Royal et la sainteté, Olivier Andurand, Philippe Luez, Éric Suire (dir.), Paris, Société des Amis de Port-Royal (Chroniques de Port-Royal; 69), 2019, p. 59-74.

50. Giacinto Arizzarra, Elementa sacrae hermeneuticae seu Institutiones ad intelligentiam, sacrarum scripturarum, Castrinovi Carfagnane, s.n., 1790. De l'ordre des frères dominicains, prieur du couvent de Fiesole, professeur à Pise et associé à l'Académie Colombaria, il fut professeur de Saintes Écritures et de langues orientales à l'université de Modène de 1772 à 1792.

51. Jacques Joseph Duguet, Règles pour l'intelligence des Saintes Écritures, Paris, J. Estienne, 1716; traduction italienne: Regole per intendere le Sante Scritture, Venise, Guglielmo Zerletti, 1775. 
le De Doctrina Christiana, joignent à l'interprétation littérale une lecture figurative de la Bible ${ }^{52}$, en s'appuyant sur une solide culture biblique, sur la connaissance des commentaires patristiques ainsi que des langues anciennes et orientales. Pour ces clercs imprégnés de jansénisme, il fallait revenir aux sources de la foi, à l'Écriture sainte et à la «simplicité» du texte biblique.

Duguet était une référence importante de leurs discours. Pujati déclare avoir pour lui une grande estime et mentionne ses lettres, notamment la troisième, dans laquelle Duguet affirme qu'il faut

[...] remonter jusqu'aux premières sources de vérités et apprendre de cet Apôtre [saint Paul] l'intelligence, et l'usage des livres de l'Ancien Testament, qui sont toujours fermés, s'il n'en donne la clef; et qui renferment toutes les promesses de la nouvelle alliance, quand il leur sert d'interprète ${ }^{53}$.

La mention de cette lettre est importante, car Duguet y synthétise aussi sa conception de la grâce, probablement partagée par Pujati.

Lanigan, quant à lui, prend explicitement Duguet comme maître sur la question du sens spirituel de l'Écriture. Lanigan connaît les interprétations médiévales, mais selon lui, il ne faut pas multiplier les sens de l'Écriture. Il suffit de les réduire à deux: le littéral et le mystique - Lanigan emploie ce terme pour désigner le sens figuratif ou le typologique. Par exemple, le sang des victimes des sacrifices de l'Ancien Testament est le type du sang du Christ. Il est permis, cependant, selon le professeur irlandais, de chercher d'autres sens mystiques, même si les Apôtres les ont ignorés. Mais, pour lui, il y a toujours un équilibre à respecter pour éviter les exagérations mystiques de Johannes Coccejus ou l'exégèse rabbinique, trop littérale, qui n'arrive pas à apprécier les vérités cachées sous l'écorce de la lettre. Il fait donc l'éloge de Duguet pour sa mesure:

Je souhaiterais que tous imitassent la prudence, et la modération du grand Duguet dans la recherche des sens spirituels des Écritures et qu'ils ne se laissassent pas aveugler par certaines apparences, comme s'il était licite de mettre des allégories partout où l'on trouve des ressemblances entre les matières exposées dans les livres saints et entre d'autres objets $[\ldots]^{54}$.

Comme Duguet, Lanigan n'hésite donc pas à affirmer que la lecture allégorique de la Bible doit être pratiquée avec une très grande précaution; en cela il rejoint les interprétations critiques les plus audacieuses, qui désapprouvaient l’allégorie;

52. Erich Auerbach, «Figura», Archivum Romanicum, n² 22, 1938, p. 436-489; réédité plusieurs fois: en français par Diane Meur et Marc de Launay, Figura. La loi juive et la promesse chrétienne, Paris, Macula, 2017.

53. Jacques Joseph Duguet, Lettres sur divers sujets de morale et de piété, Paris, J. Estienne, 1726, t. III, p. 58-59.

54. John Lanigan, Saggio..., p. 95: «Vorrei che tutti imitassero la prudenza, e la moderazione del grande Duguet nel cercare i sensi spirituali delle Scritture, e non si lasciassero abbagliare da certe apparenze, come se fosse lecito di metter allegorie, dovunque si trovino delle somiglianze tra le materie esposte nei libri sacri, e tra altri oggetti $[\ldots]$ ». 
toutefois, il rappelle que le but des Écritures est Jésus-Christ et l'Église, que Jésus est la clef pour comprendre la Bible ${ }^{55}$.

Son attaque contre le recours généralisé à l'allégorie, dans l'interprétation biblique, fruit de l'ignorance ${ }^{56}$, était partagée, d'ailleurs, par Pujati.

Cependant, aux règles déjà établies par Duguet, jugées insuffisantes ${ }^{57}$, Lanigan en ajoute d'autres plus personnelles, concernant l'explication des contradictions de l'Écriture. De plus, acquis au principe de libre examen, il risque une observation vraiment hardie, que Pujati n'osera pas proposer : "Chacun voit que les Apôtres ne sont pas des guides infaillibles qu'on doit suivre dans l'interprétation des Écritures ${ }^{58}$. L'usage que les Apôtres font de l'Écriture sainte ne constitue pas automatiquement un critère certain de vérité, puisque nous avons toujours le droit d'examiner si les explications données par les écrivains sacrés sont exactes ${ }^{59}$. Les Apôtres peuvent donc se tromper dans l'interprétation des Écritures, par conséquent, les successeurs des Apôtres le peuvent également. Cette déduction, discrètement glissée dans le texte de Lanigan, est révolutionnaire et mine, à y bien regarder, le principe établi par le Concile de Trente sur l'infaillible interprétation de l'Église concernant les Écritures. Cette affirmation de Lanigan, qui autonomise l'herméneute biblique et qui révèle sa confiance dans la raison, n'a sûrement pas échappé à Pujati qui, dans son Saggio, est toujours très attentif à rappeler les normes définies à Trente et l'obéissance que l'on doit à l'interprétation officielle de l'Église.

L'influence de Duguet se voit également dans l'introduction de son traité d'herméneutique. Il y affirme que l'étude des Saintes Écritures devrait constituer l'occupation principale des prêtres, en faisant référence à saint Jean Chrysostome, pour qui l'ignorance des Écritures est la cause de tous les maux. Lanigan dénonce les siècles d'assujettissement des Écritures à l'exégèse scolastique, qui, recourant à des citations isolées de la Bible, arrivait à des définitions dogmatiques et à des raisonnements dialectiques étrangers à la simplicité des Saintes Écritures. Lanigan considère que ce genre d'exégèse est contraire à l'esprit de l'Église primitive. Au fil des siècles les sentences de Pierre Lombard avaient ainsi pris la place de la Bible.

Cette critique de l'exégèse scolastique qui vise également les jésuites-molinistes - un autre sujet polémique caractéristique des jansénistes -, s'accompagne d'un regard positif jeté sur les études bibliques qui ont repris au $\mathrm{XVI}^{\mathrm{e}}$ siècle. Pourtant, les catholiques n'ont pas su tirer parti des remèdes donnés par les Écritures mêmes pour combattre les hérésies, à l'exemple des Pères. Alors, de faux maîtres sont apparus, dont le souci n'était pas de transmettre le sens des Écritures mais plutôt de les détruire - Lanigan vise ici les molinistes ${ }^{60}$.

55. John Lanigan, Saggio..., p. 98-99.

56. John Lanigan, Institutionum Biblicarum ..., p. XII.

57. John Lanigan, Saggio..., p. 98 sq.

58. Ibid., p. 88 .

59. Ibid.

60. John Lanigan, Institutionum Biblicarum ..., p. VII. 
Le docteur irlandais insiste donc sur l'importance de la formation des séminaristes en latin et dans les langues anciennes, en histoire et en géographie, tout en reconnaissant les progrès des études bibliques à son époque, au point que la ratio studiorum des cursus académiques prévoyait les prelectiones bibliques.

Sa défense du sens littéral est également tournée contre des rationalistes, qualifiés d' «incrédules » ${ }^{61}$, dont l'interprétation de la Bible niait le péché originel et l'historicité de la Genèse. Dans The Evidences of Natural and Revealed Religion ${ }^{62}$, Samuel Clarke ${ }^{63}$ considérait le récit biblique comme ridicule et se moquait du conte représentant le diable sous la forme d'un serpent ${ }^{64}$. De même, Johann Gottfried Eichhorn ${ }^{65}$ vidait ce récit de tout contenu théologique et l'expliquait comme une fable qui ne faisait que suivre l'antique croyance de la transition de l'âge d'or vers des temps mauvais ${ }^{66}$. Lanigan leur répond en renvoyant à Duguet ${ }^{67}$ et à Pascal ${ }^{68}$. La question du péché originel lui paraissait capitale: ceux qui le niaient osaient attribuer à Dieu le mal qui est dans le monde ${ }^{69}$. Sur ce point, Lanigan assimile les molinistes à des sociniens $\mathrm{s}^{70}$, les accusant d'attribuer le mal à Dieu par l'hypothèse d'un état de pure nature ${ }^{71}$, qui abat la religion chrétienne et ouvre la porte aux déistes. Ces derniers, malgré leur manque d'érudition biblique, se permettent de philosopher sur les livres saints. Lanigan se réfère notamment à La Bible enfin expliquée de Voltaire, ouvrage qualifié d' "exécrable »" ${ }^{72}$ que Pujati aussi mentionnera.

Dans le Saggio, le docteur irlandais estime que les sociniens restreignent le sens littéral des Écritures en cas de conflit avec la raison. Ils altèrent ainsi le sens du texte biblique, afin de mieux l'adapter à des principes rationalistes en cherchant dans

61. Ibid., p. 8-9, n. 3 et 5 .

62. Publié dans The Works of Samuel Clarke [...], Londres, J. \& P. Knapton, 1738.

63. Samuel Clarke (1675-1729), clerc anglican, fut l'un des philosophes majeurs de son temps. En 1712, il écrivit un traité sur la Trinité qui le fit considérer anti-trinitaire. En 1705, il publia les Evidences of Natural and Revealed Religion. Il étudia les Écritures dans les langues originales ainsi que les Pères de l'Église.

64. John Lanigan, Institutionum Biblicarum..., p. 7.

65. Ibid

66. Ibid., p. 8.

67. Ibid.: «Vide etiam Duguetum tentationem protoparentum egregie explicantem» [ Vois aussi comment Duguet explique parfaitement la tentation de nos ancêtres»].

68. Ibid., p. 8-9, n. 5: «Neque ipsam nostram, rerumve humanarum conditionem perspicere, aut explicare valemus, nisi ex hoc dogmate subsidium petamus» [ Nous ne pouvons pas expliquer notre condition même, si nous ne demandons pas de l'aide à ce dogme»]. "Chose étonnante», dit Pascal, «que le mystère le plus éloigné de notre connaissance, qui est celui de la transmission du péché originel soit une chose sans laquelle nous ne pouvons avoir aucune connaissance de nous-mêmes. [...] Le nœud de notre condition prend ses retours et ses replis dans cet abîme. De sorte que l'homme est plus inconcevable sans ce mystère, que ce mystère n'est inconcevable à l'homme» (Blaise Pascal, Pensées, Paris, G. Desprez, 1670, titre III, p. 38-39).

69. Ibid., p. 9-10.

70. Un groupe religieux né en Pologne vers la moitié du XVI ${ }^{e}$ siècle, qui trouva ensuite son guide en Fausto Sozzini, un théologien anti-trinitaire.

71. Voir Blaise Romeyer, «La théorie suarézienne d'un état de nature pure», Archives de philosophie, vol. 18, n 1, 1949, p. 37-63.

72. Voir John Lanigan, Institutionum Biblicarum..., p. 6, n. 2. 
les Écritures des sens différents des sens ordinaires ${ }^{73}$. Lanigan donne en exemple l'œuvre de Wolzogenius, Compendium Religionis Christianae. Les sociniens oublient les conséquences du péché originel sur la raison et méconnaissent l'insuffisance des efforts humains de compréhension sans la grâce de Jésus-Christ.

Lanigan en outre défend une méthode unique d'interprétation pour les deux Testaments. À sa suite, Pujati se bat pour la même idée:

[...] j'estime qu'il est superflu de dupliquer l'herméneutique sacrée pour les deux Testaments de l'Écriture, parce que pour interpréter l'un et l'autre les mêmes règles sont nécessaires, les mêmes principes et les mêmes connaissances; d'autant plus que l'Ancien Testament porte en son sein le Nouveau et il en est comme une longue allégorie ou une parabole, sans nuire aucunement à la vérité de l'histoire; et réciproquement, le Nouveau Testament éclaire l'Ancien et il est la vérité de la figure, ou le corps de l'ombre ${ }^{74}$.

Le choix d'une seule herméneutique pour l'ensemble des Écritures est lié à la promotion d'une exégèse que l'on peut qualifier de «canonique». Lanigan est favorable à ce qu'un passage soit éclairé par d'autres versets ou d'autres péricopes puisque le canon des Écritures les unit. Il considère cette pratique interprétative comme un sommet de l'exégèse:

[...] l'ensemble des Écritures est semblable à un beau corps, dont les membres sont tellement bien proportionnés entre eux, qu'ils se soutiennent les uns les autres; ils ne peuvent être séparés les uns des autres, sans préjudice pour leur merveilleuse cohésion, par laquelle Dieu les a unis. Le grand art d'expliquer l'Écriture par l'Écriture consiste à montrer comment les parties de ce bel ensemble se soutiennent les unes les autres; il faut un esprit pénétrant et une grande connaissance des livres sacrés pour posséder pleinement cet $\operatorname{art}^{75}$.

Pour Lanigan, cette méthode permet de résoudre les nombreuses contradictions apparentes de l'Écriture. Le docteur irlandais préconise de prendre pour règle de foi le sens le plus simple et d'expliquer les passages plus complexes à partir du plus simple. Cette méthode permet aussi de limiter le recours à l'interprétation allégorique, qui ne s'impose que lorsqu'aucune lecture conciliatrice n'est possible.

73. John Lanigan, Saggio..., p. 57.

74. Giuseppe Maria Pujati, Saggio di ermeneutica sacra, p. 6-7: «[...] Io m'avviso essere superfluo il duplicare l'Ermeneutica sacra pei due Testamenti della Scrittura. Imperciocchè ad interpretare l'uno e l'altro si richiedono le stesse regole, gli stessi principi e le stesse cognizioni: tanto più che il Testamento antico si porta come in seno il nuovo e n'è come una lunga allegoria o parabola, senza nuocere punto alla verità della storia; e reciprocamente il nuovo illumina il vecchio ed è la verità della figura o il corpo dell'ombra».

75. John Lanigan, Saggio..., p. 129-130: «[...] il complesso delle Scritture è simile ad un bel corpo, le di cui membra sono cosi proporzionate tra di loro, che si sostengono vicendevolmente e non si possono distaccare le une dalle altre, senza pregiudicare alla maravigliosa connessione, colla quale Dio le ha unite. Nello scambievole avvicinamento delle parti di questo bel tutto, e nel far vedere come si sostengano reciprocamente, consiste la grand'arte di spiegare la Scrittura colla Scrittura istessa; arte, per possedere la quale a fondo ci vuole un genio penetrante, e una gran cognizione dei libri sacri». 
L'exégèse canonique préconisée par Lanigan est fondée sur la conviction que Jésus-Christ est à la fois l'objet de toute l'Écriture, mais aussi son interprète. Puisqu'il n'a pas laissé de système herméneutique ${ }^{76}$, il revient à ses disciples de réfléchir sur la manière dont il a interprété les Écritures et d'en tirer des normes précises. Il faut donc suivre une sorte de méthode inductive, à rebours de la méthode scolastique: examiner l'exemple concret de Jésus pour en tirer les principes. Ensuite, à partir de ces derniers, l'interprète a le droit de tout examiner et de tout vérifier, quitte à s'éloigner de l'interprétation des Apôtres.

Lanigan décrit cet art d'interpréter les Écritures à la suite de Jésus-Christ comme un don du Saint-Esprit pour accomplir la mission d'enseigner aux hommes le sens véritable des Écritures ${ }^{77}$, don qui n'est autre que celui promis par JésusChrist à ses disciples ${ }^{78}$. Cette conception charismatique de l'exégèse fait du travail philologique une condition nécessaire mais non suffisante. Comme le théologien hébraïsant Johann David Michaelis (1717-1791), Lanigan accorde une grande importance aux sciences profanes pour éclairer les obscurités du sens littéral, car les textes sacrés ont à ses yeux un caractère historique qui doit être soumis à un examen scientifique. Cependant, privilégier la philologie au détriment du sens théologique et spirituel du texte équivaut à faire du rabbinisme et peut nuire à la compréhension des intentions véritables et du but des auteurs bibliques: conduire aux vérités annoncées par les prophètes et aux réalisations des prédictions bibliques dans le Christ et dans l'Église. L'érudition ne suffit pas pour interpréter les Écritures.

Nous avons donc vu que Lanigan touche à des sujets jansénistes - le figurisme, la conversion des Juifs, la lutte contre les jésuites-molinistes et contre les sociniens, une conception charismatique de l'exégèse, le primat du sens théologique et spirituel du texte - et se laisse inspirer par Duguet.

\section{Conclusion}

L'herméneutique proposée par Lanigan se situe au croisement de deux traditions interprétatives qui s'étaient souvent affrontées à la fin du XVII ${ }^{e}$ et au cours du XVIII ${ }^{e}$ siècle: d'une part, l'exégèse critique qui entend soumettre la Bible aux sciences du texte; d'autre part, la prolongation d'une quête d'un sens spirituel des Écritures. En effet, Lanigan entendait se battre sur deux fronts: d'un côté, la défense de l'érudition biblique contre l'ignorance et les abus allégoriques; de l'autre, la défense de l'inspiration des Écritures contre les attaques rationalistes. Il opère sa synthèse dans le cadre d'un projet de réforme du clergé assez caractéristique du jansénisme italien de son époque. Au-delà de son attachement aux règles de Duguet, on peut mesurer l'écart de son herméneutique avec celle de Port-Royal: l'autorité de la tradition apostolique et patristique cède le pas au libre examen de

\footnotetext{
76. Ibid., p. 86.

77. Ibid., p. 86-87.

78. Ibid., p. 87.
} 
l'exégète, doublement pourvu par ses compétences scientifiques et son charisme. Le don d'interpréter les Écritures est déplacé de l'Église enseignante tout entière à l'interprète lui-même.

La multiplicité de sens émerge ainsi selon Lanigan chez l'interprète éclairé par un don surnaturel, et non pas dans l'«objet» interprété.

Nous pouvons aussi remarquer que Lanigan valorise un concept qui sera le fondement de l'herméneutique de Schleiermacher, à savoir que la compréhension d'une œuvre consiste dans la pénétration psychologique des idées qui ont guidé son auteur.

L'objection que Lanigan adresse enfin aux excès de la méthode historico-critique n'est pas différente de celle de Dilthey et Gadamer, « disciples » de Schleiermacher, avec leur distinction entre sciences de la nature et sciences de l'esprit.

Grazia Grasso

Laboratoire d'études sur les monothéismes (LEM) École pratique des hautes études (EPHE) 